\title{
A GEOMAGNETIC FIELD SPECTRUM
}

\author{
R. A. Langel \\ Goddard Space Flight Center, Greenbelt, Maryland 20771 \\ R. H. Estes
}

Business and Technological Systems, Seabrook, Maryland 20706

Abstract. Selected MAGSAT data are used to derive a spherical harmonic model of the earth's internal magnetic field of degree and order 23. The power spectrum, with terms of the form (Mauersberger, 1956; Lowes, 1974):

$$
R_{n}=(n+1) \sum_{m=0}^{n}\left(\left(g_{n}^{m}\right)^{2}+\left(h_{n}^{m}\right)^{2}\right)
$$

is computed. It exhibits a distinct change of slope at about $\mathrm{n}=14$ which is interpreted to mean that the field from the core dominates for $n \leqslant 13$ and the field from the crust for $n \geqslant 15$. The two portions of the spectrum are well represented by: $R_{n}=1.349 \times 10^{9}(0.270)^{n}(n T)^{2}$ for the core and $R_{n}=37.1(0.974)^{n}(n T)^{2}$ for the crust. These representations can be used to establish order of magnitude inaccuracies in core field models due to crustal fields and due to inability to "observe" the core field wavelengths beyond $\mathrm{n}=13$ which are totally obscured by the crustal field.

\section{Introduction}

As shown by Gauss in 1839 , the potential of the internal geomagnetic field can be represented by a spherical harmonic analysis of the form:

$$
\begin{aligned}
V & =a \sum_{n=1}^{N} \sum_{m=0}^{n}\left(\frac{a}{r}\right)^{n+1}\left[g_{n}^{m} \cos m \phi\right. \\
& \left.+h_{n}^{m} \sin m \phi\right] P_{n}^{m}(\cos \theta)
\end{aligned}
$$

where: $a$ is the mean radius of the earth,

$$
\begin{aligned}
& \mathrm{r}, \theta, \phi \text { are the standard spherical coordinates, and } \\
& \mathrm{P}_{\mathrm{n}}^{\mathrm{m}} \text { (in "modern" methodology) are the Schmidt quasi- } \\
& \text { normalized form of associated Legendre functions. }
\end{aligned}
$$

The magnetic field is then given by:

$$
\overline{\mathrm{B}}=-\nabla \mathrm{V}
$$

Measured data are used to derive the coefficients $g_{n}^{m}$ and $h_{n}^{m}$, usually by some form of least squares procedure.

The measured magnetic field contains contributions from sources within the earth's core and crust, from electric currents in the lower mantle, and from external currents in the ionosphere and magnetosphere. Assuming that any effects of the external currents can be minimized by proper data selection and processing in a global data set, the coefficients in (1) can be solved to determine a model for the combined effects of the core and crustal fields. Further, one would expect the low degree and order terms to be dominated by the field from the earth's core and the higher degree and order terms to be domi-

Copyright 1982 by the American Geophysical Union.

Paper number 2L0113.

0094-8276/82/002L-0113\$3.00 nated by the field from the earth's crust (see, e.g. Alldredge et al., 1963; Bullard, 1967). This paper describes an attempt to construct a suitable data set from Magsat data and determine the degree at which the dominance passes from core to crustal sources.

\section{The Data Set}

The data consist of 26,500 Magsat scalar and vector data points taken on fourteen selected magnetically quiet days over the interval November 5, 1979 to March 15, 1980. Vector data were limited to $\pm 50^{\circ}$ latitude so as to reduce the contamination due to field aligned currents. The vector data were corrected for attitude biases and external fields based on field models derived separately for the individual fourteen days, as described by Langel et al (1981). A linear secular variation model to degree and order seven, computed from the Magsat data, was used to reduce these data to epoch 1980.0. Table 1 gives the days from which the data were selected, the number of observations and the measurement standard deviations used in the least squares adjustment. The standard deviations represent the standard deviations of fits of the data to separate, individual field models based on each of the quiet days. Langel et al (1982) discuss how the measurement standard deviations are used to weight the data in the least squares estimation algorithm.

\section{The Model}

Derivation of the model was a two step process. In the first step all coefficients were allowed to adjust freely in the least squares estimation. At this point the standard error of each coefficient was examined and the ratio of the coefficient to its standard error was calculated. In the second step, the field model determined in the first step was used as the a priori estimate of the field, except that those coefficients whose ratio was less than 2.5 (in the first step) were constained to be zero in the step two least squares adjustment. This criteria for eliminating coefficients which are poorly determined (a ratio greater than or equal to 2.5 implies the coefficient has statistical significance above the $99 \%$ confidence level) is similar to that used by Barraclough and Malin (1979) for modeling secular acceleration. The derived coefficients, designated MGST(10/81), and their standard errors are available from the authors. For most purposes lower degree and order models, such as MGST(6/80) (Langel et al., 1980) or GSFC(9/80) (Langel et al., 1982) are more suitable.

\section{The Spectrum}

The primary purpose for deriving MGST(10/81) was to examine its power spectrum. The form of that spectrum is taken to be the one introduced by Mauersberger (1956) and by Lowes $(1966,1974)$ where: 
TABLE 1. Magsat Scalar and Vector Data Used in Model

\begin{tabular}{|c|c|c|}
\hline Date & $\begin{array}{c}\text { Number of } \\
\text { Observations } \\
(B, X, Y, Z)\end{array}$ & $\begin{array}{l}\text { Data Sigma (nT.) } \\
\text { Used for Weighting }\end{array}$ \\
\hline $\begin{array}{lr}\text { Nov. } & 5,1979 \\
\text { Nov. } & 21,1979 \\
\text { Nov. } & 22,1979 \\
\text { Dec. } & 13,1979 \\
\text { Dec. } & 25,1979 \\
\text { Jan. } & 9,1980 \\
\text { Jan. } & 10,1980 \\
\text { Jan. } & 18,1980 \\
\text { Jan. } & 19,1980 \\
\text { Feb. } & 12,1980 \\
\text { Feb. } & 13,1980 \\
\text { Mar. } & 2,1980 \\
\text { Mar. } & 3,1980 \\
\text { Mar. } & 15,1980\end{array}$ & $\begin{array}{l}1,500 \\
1,200 \\
1,200 \\
2,800 \\
2,700 \\
1,200 \\
1,200 \\
1,600 \\
1,600 \\
2,100 \\
2,100 \\
2,400 \\
2,400 \\
2,500 \\
\end{array}$ & $\begin{array}{r}10 \\
15 \\
15 \\
10 \\
10 \\
20 \\
20 \\
15 \\
15 \\
10 \\
10 \\
10 \\
10 \\
5\end{array}$ \\
\hline & $\overline{26,500}$ & \\
\hline
\end{tabular}

$$
R_{n}=(n+1) \sum_{m=0}^{n}\left[\left(g_{n}^{m}\right)^{2}+\left(h_{n}^{m}\right)^{2}\right]
$$

$R_{n}$ is the mean square value over the earth's surface of the magnetic field intensity produced by harmonics of the $n$th degree. The set $\left[R_{n}\right]$ is tabulated in Table 2 and plotted in Figure 1. The dipole term obviously stands alone, and a break in the spectrum is apparent near degree 14. This is in agreement with earlier results of Cain et al (1974) and Cain (1976). Our interpretation of Figure 1 is that the core field dominates for $n \leqslant 13$, and the crustal field for $n \geqslant 15$.

This interpretation is supported by the analysis of McLeod and Coleman (1980) who derived the expected shape of the spherical harmonic power spectrum, $R_{n}$, using a statistical model for the core and crustal fields. Their theoretical spectrum is in close agreement with Figure 1 in shape although their crustal spectrum is about a factor of three lower in amplitude.

The straight lines on Figure 1 are linear regressions to $R_{\mathfrak{n}}$ for $\mathrm{n}=2$ to 12 and 16 to 23 . They are:

$$
R_{n}=1.349 \times 10^{9}(0.270)^{n}(n T)^{2},
$$

TABLE 2. The Geomagnetic Spectrum From MGST(10/81)

\begin{tabular}{rccr}
\hline$n$ & $R_{n}(n T)^{2}$ & $n$ & $R_{n}(n T)^{2}$ \\
\hline 1 & $1.869 \times 10^{9}( \pm 0.05)$ & 13 & $104.86( \pm 1.18)$ \\
2 & $6.148 \times 10^{7}( \pm 0.10)$ & 14 & $37.40( \pm 1.33)$ \\
3 & $3.562 \times 10^{7}( \pm 0.15)$ & 15 & $31.51( \pm 1.27)$ \\
4 & $1.033 \times 10^{7}( \pm 0.23)$ & 16 & $27.64( \pm 1.17)$ \\
5 & $2.032 \times 10^{6}( \pm 0.32)$ & 17 & $22.84( \pm 1.12)$ \\
6 & $5.114 \times 10^{5}( \pm 0.43)$ & 18 & $24.78( \pm 1.59)$ \\
7 & $1.456 \times 10^{5}( \pm 0.56)$ & 19 & $14.26( \pm 0.79)$ \\
8 & $1.979 \times 10^{4}( \pm 0.71)$ & 20 & $22.67( \pm 1.49)$ \\
9 & $1.509 \times 10^{4}( \pm 0.89)$ & 21 & $13.84( \pm 1.33)$ \\
10 & $2.523 \times 10^{3}( \pm 1.07)$ & 22 & $22.84( \pm 1.15)$ \\
11 & $8.057 \times 10^{2}( \pm 1.24)$ & 23 & $32.46( \pm 2.00)$ \\
12 & $1.975 \times 10^{2}( \pm 1.22)$ & & \\
\hline
\end{tabular}

for the core and

$$
R_{n}=37.1(0.974)^{n}(n T)^{2} \text {, }
$$

for the crust,

\section{Discussion}

If our interpretation of Figure 1 is correct, then the turn in the spectrum does not reflect a limitation of the data but rather the separate field sources, and the higher degree and order spherical harmonic coefficients, while not as well determined as the lower degree/order terms, nonetheless are physically meaningful.

Practically speaking, this means that the presence of crustal fields places a limitation on our ability to estimate the field from the earth's core. In particular, the accuracy of terms of a given degree for describing the core field will depend upon the relative amplitude of the core and crustal components for that degree. These can be estimated from Figure 1. Models derived using these techniques should not extend beyond degree/order 14 if they are intended to represent only the core field. New techniques, perhaps such as that developed by Shure et al (1981), will be required to estimate core fields at higher degree and order. It should also be noted that Figure 1 implies limitations on terms of high degree/order, yet less than fourteen. For example one should not expect to determine terms of degree/order twelve and thirteen to within $1 \%$ since more than $1 \%$ of the contribution at these wavelengths is crustal in origin.

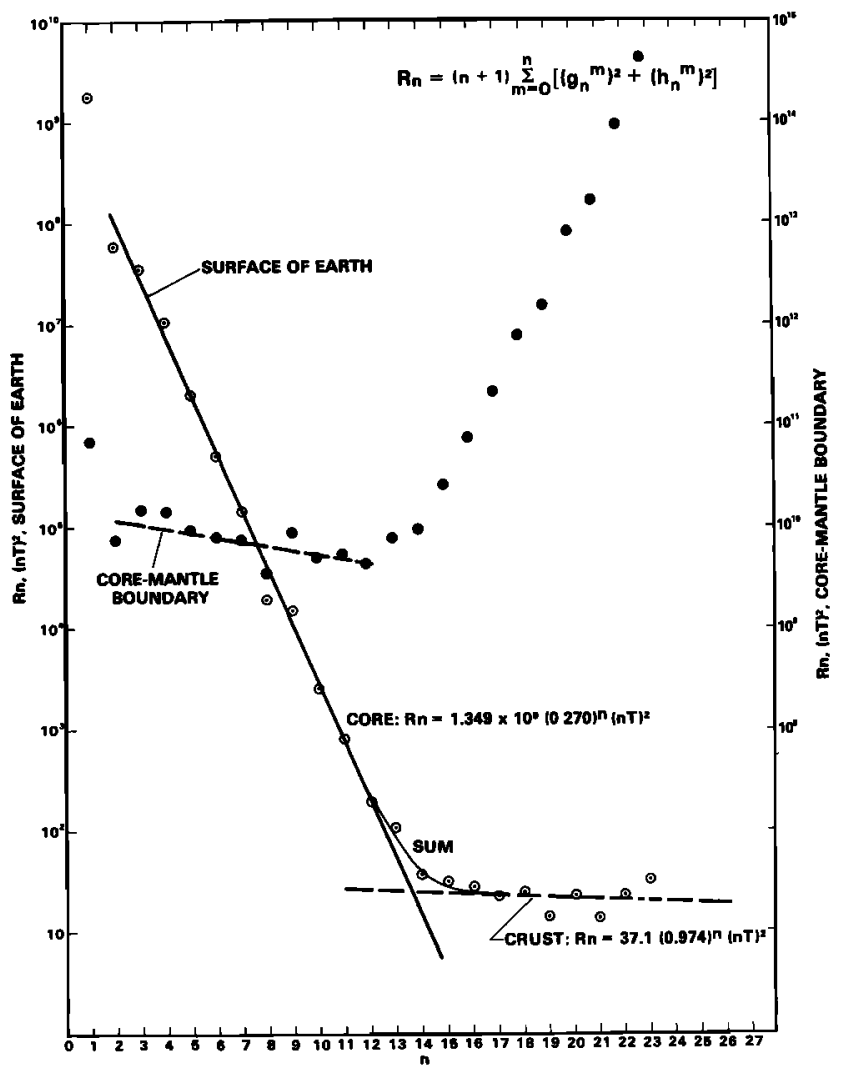

Fig. 1. Geomagnetic field spectum. $R_{n}$ is the total mean square contribution to the vector field by all harmonics of degree $n$. The curves are fit to the surface result and extrapolated to the core-mantle boundary. 


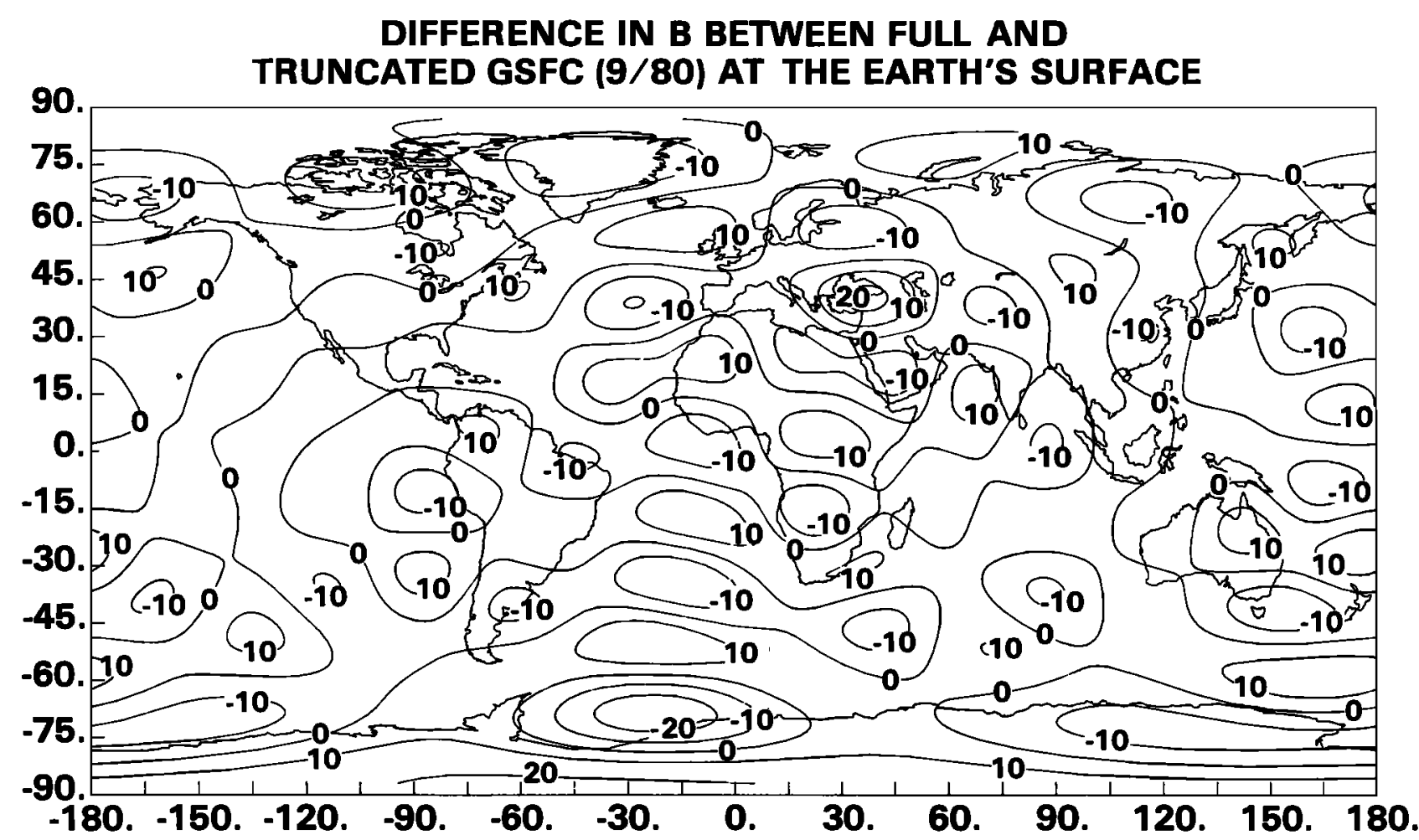

Fig. 2. Difference in scalar field between the full (degree $=13) \mathrm{GSFC}(9 / 80)$ model and the GSFC(9/80) model truncated to degree ten, at the earth's surface. Units are nT.

One of the applications of spherical harmonic magnetic field models is to estimate the main field contribution in aeromagnetic surveys so that it can be removed to isolate the anomalous field originating in the crust. Our results imply that models used for that purpose should extend to at least degree/order thirteen. This, of course, is more important for isolating anomalies at satellite altitude, where the anomaly amplitude is very small $(0-50 \mathrm{nT})$, than at the surface. To provide an estimate of the importance of higher degree/ order terms, we compared two versions of the GSFC (9/80) field model (Langel et al, 1982). One version was the full degree/order thirteen model and the other a truncation to degree/order ten. Figure 2 shows the difference in field magnitude at the earth's surface. The differences are up to $20 \mathrm{nT}$ in magnitude and between $10^{\circ}-20^{\circ}$ in wavelength. Thus, if it is necessary to isolate anomaly fields with an accuracy better than $20 \mathrm{nT}$, the full thirteenth degree/order model is required.

Because of its definition, $R_{n}$ can be used to estimate the magnitude of the field due to terms of specific degree(s). For example, the rms core field of degree eleven is given by

$$
\sqrt{R_{11}}=\sqrt{1.349 \times 10^{9}(0.270)^{11}}=27.4 \mathrm{nT},
$$

where the expression for $R_{11}$ is taken from equation (4). The argument may be extended to estimate the difference in accuracy of models of degree ten and thirteen:

$$
\sqrt{R_{11}+R_{12}+R_{13}}=31.7 \mathrm{nT},
$$

in fair agreement with Figure 2. We can also estimate the inaccuracies in core field models resulting from not being able to determine terms above degree thirteen by evaluating the root of $\sum_{n=14}^{\infty} R_{n}$, where $R_{n}$ is calculated from equation (4).
The evaluation gives $4.50 \mathrm{nT}$ which then may be regarded as an upper bound on the absolute accuracy with which we can determine the magnitude of crustal anomalies.

Lowes' (1974, expression 4) equation for $R_{n}$ is:

$$
R_{n}=4.0 \times 10^{10}(0.222)^{n}(n T)^{2} .
$$

This was derived from the $n=3$ to 8 terms of IGRF 1965.0 The slope of (8) is steeper than that of (4) so that (8) underestimates the contribution of the higher degree terms. Examination of our Figure 1 and Lowes' Figure 2 shows that both the $n=2$ and $n=8$ terms are below the computed lines in both analyses. Thus the omission by Lowes of the $n=2$ term, together with the non-availability of terms beyond $\mathrm{n}=8$, resulted in the steeper slope.

The fact that the higher order terms are more important than previously thought affects our estimate of the accuracy to which we know the field at the core-mantle boundary. $R_{n}$, and its standard error, may be extrapolated to the coremantle boundary, assuming that the effects of mantle currents

TABLE 3. The rms field and its Standard Error at the Core-Mantle Boundary

\begin{tabular}{ccc}
\hline$n$ & rms field $(\mathrm{nT})$ & $\operatorname{rms} \sigma(\mathrm{nT})$ \\
\hline 1 & $2.64 \times 10^{5}$ & 1.4 \\
$2-12$ & $2.93 \times 10^{5}$ & $6.1 \times 10^{3}$ \\
$1-12$ & $3.94 \times 10^{5}$ & $6.1 \times 10^{3}$ \\
$\geqslant 2$ & $3.56 \times 10^{5}$ & $\left(2.03 \times 10^{5}\right)$ \\
$>12$ & $2.03 \times 10^{5}$ & $\left(2.03 \times 10^{5}\right)$ \\
Total & $4.43 \times 10^{5}$ & $\left(2.03 \times 10^{5}\right)$ \\
\hline
\end{tabular}


are negligible, by multiplying by the factor $(a / r)^{2 n+4}$, where $a=6372.1 \mathrm{~km}$ and $\mathrm{r}$ is taken to be the seismic boundary at $3485 \mathrm{~km}$. Figure 1 shows the transformed values of $R_{n}$ and the line corresponding to the transformed equation (4), which is:

$$
R_{n}(r=3485 \mathrm{~km})=1.51 \times 10^{10}(0.903)^{\mathrm{n}}(\mathrm{nT})^{2}
$$

The core field portion of the spectrum is now almost level, i.e. the higher harmonics, which are less well determined, are relatively more important that at the surface. The radius at which the transformed equation (4) gives a "white" spectrum is $r=3311 \mathrm{~km}$, which is $174 \mathrm{~km}$ below the seismic coremantle boundary.

In our interpretation, crustal fields totally dominate $\mathbf{R}_{\mathrm{n}}$ for $n \geqslant 15$ and significantly contribute to $R_{n}$ for $n=13$ and 14. While the contribution of these higher degree terms is very small at the earth's surface relative to the total field magnitude, at the core-mantle boundary a large portion of the field is contained in the high degree terms. Following Lowes (1974), we can use $R_{n}$ from equation (9) to estimate the rms contribution from various field harmonics. The dipole term and standard errors are estimated by transforming the values directly from Table 2 . Table 3 summarizes these results.

The Table indicates that the total rms field at the coremantle boundary is $4.43 \times 10^{5} \mathrm{nT}$. Note that the estimate of the rms field above degree twelve is $2.03 \times 10^{5}$, about 57 percent of the total non-dipole field and 46 percent of the total field. Unfortunately the terms of degree thirteen and beyond are essentially unknown because of the dominance of the crustal fields. This means that the standard error for $n>$ 12 is equal to the rms field value (standard errors so estimated are enclosed in parenthesis in Table 3), and the conclusion is that the percentages given also reflect the uncertainty of our knowledge of the field at the core-mantle boundary. These percentages are much higher than those derived by Lowes (1974).

It is perhaps worth noting that in several cases the deviations of the points from the line in Figure 1 are much greater than would be expected from the error estimates. The degree eight term is the most obvious of these. In fact, this term is low in the spectra of models derived solely from POGO satellite data and solely from surface data as well as from MAGSAT data. This might indicate that real features are reflected in the structure of the spectrum.
Acknowledgements. The authors wish to acknowledge valuable discussion with G.D. Mead and E.R. Lancaster of Goddard Space Flight Center and the fine efforts of the MAGSAT project under the direction of J. Murphy (NASA Headquarters), G.M. Ousley (GSFC) and L.D. Eckard (APL).

\section{References}

Alldredge, L.R., G.D. Van Vooris and T.M. Davis, A magnetic profile around the world, J. Geophys. Res., 68, 3679-3692, 1963.

Barraclough, D.R., and S.R.C. Malin, Geomagnetic secular acceleration, Geophys. J.R. astr. Soc., 58, 785-793, 1979.

Bullard, E.C., The removal of trend from magnetic surveys, Earth Planet Sci Letters, 2, 293-300, 1967.

Cain, J.C., Introductory remarks (abstract), EOS, Trans. A.G.U., 57, 907, 1976.

Cain, J.C., W.M. Davis and R.D. Regan, An N = 22 model of the geomagnetic field (abstract), EOS, Trans. A.G.U., 56, $1108,1974$.

Langel, R., R.H. Estes, G.D. Mead, E.B. Fabiano and E.R. Lancaster, Initial geomagnetic field model from MAGSAT vector data, Geophys. Res. Let., 7, 793-796, 1980.

Langel, R., J. Berbert, T. Jennings and R. Horner, Magsat data processing: A report for investigators, GSFC Report TM82160, November 1981.

Langel, R.A., R.H. Estes and G.D Mead, Some new methods in geomagnetic field modeling applied to the 1960-1980 epoch, Submitted to Jour. Geomag. Geoelec., 1982.

Lowes, F.J., Mean square values on the sphere of spherical harmonic vector fields, J. Geophys. Res., 71, 2179, 1966.

Lowes, F.J., Spatial power spectrum of the main geomagnetic field, and extrapolation to the core, Geophys. J.R. astr. Soc., 36, 717-730, 1974.

Mauersberger, P., Das mittel der energiedichte des geomagnetischen hauptfeldes an der erdoberfläche und seine säkulare, Anderung, Gerlands Beitr. Geophys., 65, 207215, 1956.

McLeod, M.G. and P.J. Coleman, Jr., Spatial power spectra of the crustal geomagnetic field and core geomagnetic field, Phys. of the Earth and Planet Inter., 23, 5-19, 1980.

Shure, L., R.L. Parker and G.E. Backus, Harmonic splines for geomagnetic modeling, Submitted to Physics of the Earth and Planetary Interiors, 1981.

(Recelved November 17, 1981; accepted January 5, 1982.) 